\title{
Genetic Algorithm Modeling for Photocatalytic Elimination of Impurity in Wastewater
}

\author{
Raheleh Jafari ${ }^{1}$, Sina Razvarz ${ }^{2}$, Wen $\mathrm{Yu}^{2}$, Alexander Gegov ${ }^{3}$, Morten \\ Goodwin $^{1}$, and Mo Adda ${ }^{3}$ \\ 1 Centre for Artificial Intelligence Research (CAIR), University of Agder, Grimstad, \\ Norway \\ 2 Departamento de Control Automático, CINVESTAV-IPN (National Polytechnic \\ Institute), Mexico City, Mexico \\ 3 School of Computing, University of Portsmouth, Buckingham Building, \\ Portsmouth PO1 3HE, UK \\ raheleh.jafari@uia.no
}

\begin{abstract}
The existence of C.I. Acid Yellow 23 (AY23) in water causes a great danger to people and society. Here, we suggest an advanced technique which predicts the photochemical deletion of AY23. The genetic algorithm (GA) technique is suggested in order to predict the photocatalytic removal of AY23 by implementing the $\mathrm{Ag}-\mathrm{TiO}_{2}$ nanoparticles provided under appropriate conditions.

In order to evaluate the proposed method, a total of 100 data are utilized which are arbitrarily divided into two: 80 samples in order to train the model as well as 20 samples in order to test the model. Experimental outcomes reveals that the suggested technique is efficient for photocatalytic elimination of impurity in wastewater.
\end{abstract}

Keywords: C.I. Acid Yellow 23, Ag- $\mathrm{TiO}_{2}$, genetic algorithm, wastewater

\section{Introduction}

Some countries all over the world encounter with a crucial problem of water impurity caused by dyes. Synthetic dye is considered as a main water pollutant as well as industrial contaminant. Dyes are utilized with different industries in order to color their products. Various investigations are carried on in order to demonstrate that dyes are toxic or carcinogenic. In a case that these colorants interact with some specific drugs inside the body of the people, they can create serious allergic and asthmatic reactions for whom they are sensitive [1]. The other serious problem generated by these dyes is that it is impossible to remove them by traditional water treatment systems. Therefore, the removal of dyes from wastewater is considered as a crucial issue for the relevant industries.

Resolving real-world problems by means of conventional optimization techniques is highly difficult. Since 1960 a great interest is developed between researchers in order to resolve complex optimization problems. Artificial neural 
network is considered as an effective tools which is highly capable in modeling complicated systems [2-10]. Boosted regression trees as well as artificial neural networks are implemented in order to model and optimize the methylene blue as well as $\mathrm{Cd}(\mathrm{II})$ elimination in [11]. The simulation of natural evolutionary procedures associated with the human beings results in stochastic optimization techniques and is named as evolutionary algorithms (EAs). EAs have high performance in comparison with conventional optimization methods which are used in order to deal with the real-world problems. Researchers generally implicate metaheuristic optimization algorithms to be mentioned as genetic algorithm (GA), particle swarm optimization (PSO), and imperialist competitive algorithm (ICA).

PSO is considered as a searching algorithm which is implemented in order to search large as well as non-linear spaces, in a case that the knowledge of researchers is limited and also the traditional optimization techniques are not qualified [12-15]. PSO is an efficient evolutionary algorithm with the global optimization ability $[16,17]$. For evaluation and finding the significant candidates for a task, the ICA technique is proposed which performs so good [18-21]. It is not required for ICA algorithm to have so precise stated mathematical model. The same with other evolutionary kinds, this algorithm starts with an initial population. Population individuals named as country are made of two parts, colonies and imperialists where all together construct some empires [22]. GA is effectively utilized to various problems because of its universal outlook, simpleness and inherent parallel processing [23-25]. It is constructed from the evolutionary nature of the human genes. GA utilizes fitness functions which have connection with criteria for giving grades to the candidates. Furthermore, this algorithm uses evolutionary techniques such as crossover and mutation on chromosomes or strands of information in order to obtain the superior examples from a great region of probabilities.

In this paper the GA technique is implemented which predicts the photocatalytic deletion of $\mathrm{AY} 23$ utilizing $\mathrm{Ag}-\mathrm{TiO}_{2}$ nanoparticles provided under appropriate conditions. This paper is based on the real-life multi-stage case with 100 data set obtained through a statistical design associated with the study. The model is built based on the artificial neural network where GA approach is utilized in order to train it. Four parameters such as initial dye concentration, UV light intensity, initial dosage of nano $\mathrm{Ag}-\mathrm{TiO}_{2}$ and irradiation time are inputs and deletion of AY23 is output. The experimental results demonstrate that the GA approach performs efficiently. Furthermore, the analysis outcomes shows that AY23 primary concentration is the most important factor, which has great impact on the AY23 elimination. The work is organized as follows. In Section 2 , the technique utilized in this paper is described. The result are analyzed in Section 3. Section 4 concludes the work and provides discussions on further work. 


\section{Proposed technique}

\section{$2.1 \quad$ Substances}

Tetraisoprpylorthotitanate $\mathrm{Ti}\left(\mathrm{OC}_{3} \mathrm{H}_{7}\right)_{4}$, methanol $(\mathrm{MeOH})$ and also silver nitrate $\left(\mathrm{AgNO}_{3}\right)$ are produced in Merck (Germany) and implemented without any extra filtration. Acid Yellow 23 is produced in Acros (USA) and used without extra filtration. The chemical structure of the Acid Yellow 23 is presented in Fig. 1. Deionized water is implemented in this experiment.

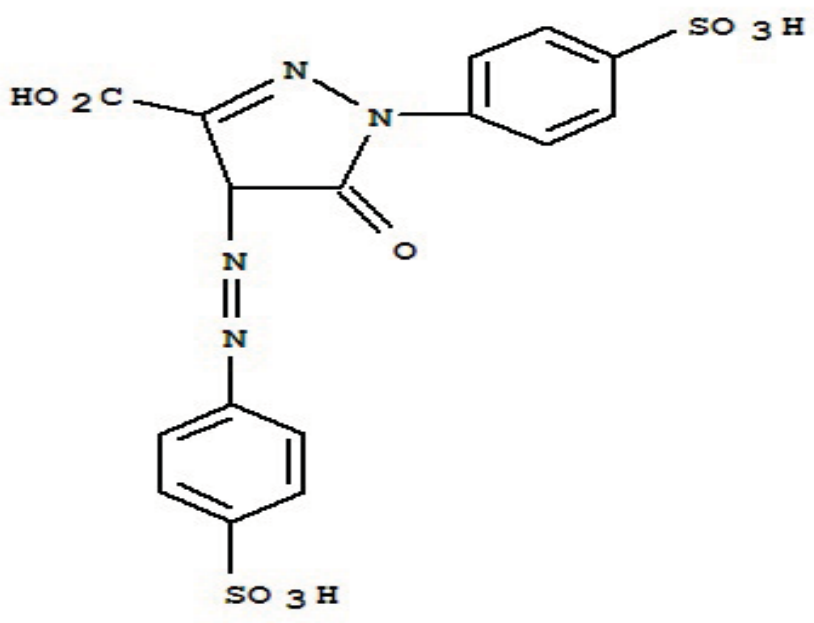

Fig.1. Chemical structure of the Acid Yellow 23.

\subsection{Ultrasonic bath $(\mathrm{T} 460 / \mathrm{H})$}

The ultrasonic bath Elma $(\mathrm{GmbH})$ is implemented having the operating frequency of $34 \mathrm{KHz}$ and a rate output power of $169 \mathrm{~W}$. The dimensions of the bath is $239 \mathrm{~mm} \times 136 \mathrm{~mm} \times 99 \mathrm{~mm}$. The whole inner formation is made of stainless steel.

\subsection{Genetic algorithm model}

GA is considered as a stochastic search algorithm based on the the mechanism related to the natural selections as well as natural genetics [26]. It is totally different from conventional search methods. GA initiates with a preliminary set of arbitrary solutions which is named as population satisfying boundary. Every individual in the population is called chromosome that displays a solution associated with the problem. The chromosome is produced by means of successive iterations which are named as generations. Via every generation, the chromosomes are investigated by means of some methods of fitness. In order to produce the next generation, the new chromosomes which are names as offspring are 
generated via either combining two chromosomes from latter generation and implementing a crossover operator or modifying a chromosome by means of a mutation operator. Fittest chromosomes have high probabilities in order to get chosen. After some generations, the algorithms approaches to the best chromosome which states the optimum or suboptimal solution associated with the problem [27]. Fig. 2 demonstrates a flowchart of a general genetic algorithm. The proposed GA matches with the general form discussed above.

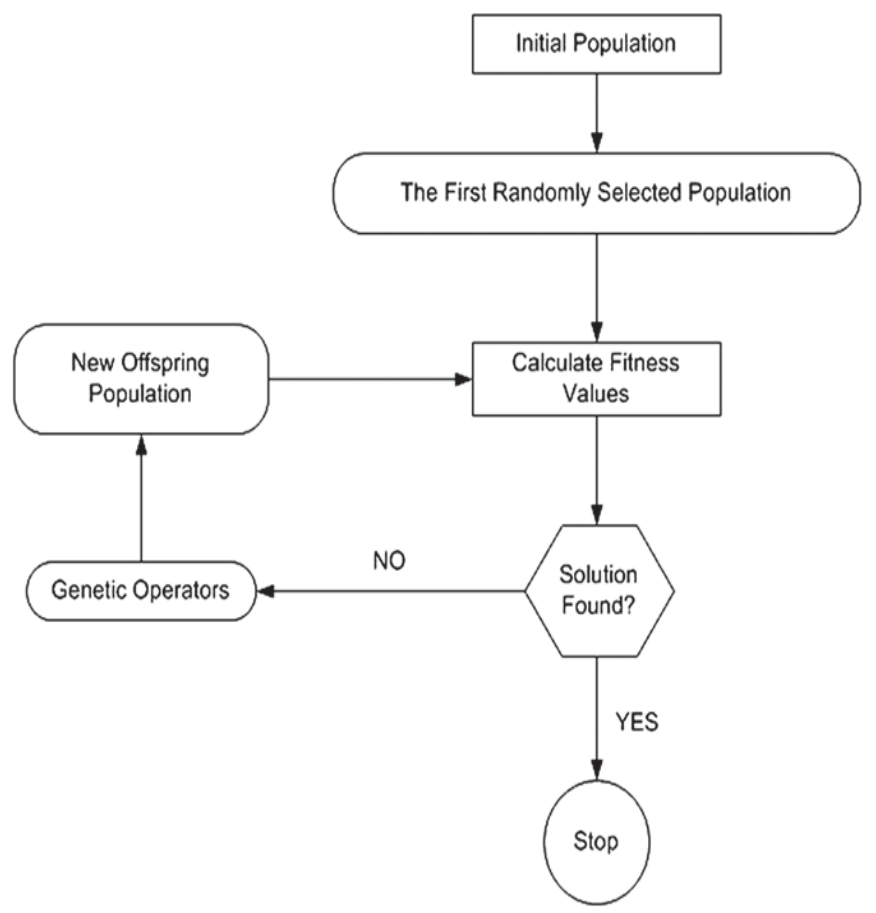

Fig.2. The flowchart of a general genetic algorithm.

\subsection{The dataset}

The aim of this paper is to generate artificial intelligence concept based on the predictive model in order to delete the AY23 in water by using a set of selected variables named as the estimators. The implemented dataset in this paper for generating the GA model is on the basis of the laboratory investigations carried out under statistical experimental design. In order to evaluate the proposed method, a total of 100 data are utilized which are arbitrarily divided into two: 80 samples in order to train the model as well as 20 samples in order to test the model.

\section{Result analysis}

Recently, artificial intelligence is taken more attraction because of its application in various fields [28-39]. GA is considered as a class of the artificial intelligence 
technique which contains a high performance in the literature. The mean square error (MSE) is used for analyzing the efficiency of the GA technique for omitting the AY23 in water. MSE can be computed from the predicted model and actual calculated values associated with the response variable as mentioned below,

$$
M S E=1 / M \sum_{i=1}^{M}\left(\widetilde{A}_{i}-\widetilde{Y}_{i}\right)^{2}
$$

such that $\widetilde{A}_{i}$ and $\widetilde{Y}_{i}$ are taken to be the predicted model as well as calculated values of the response variable, respectively. $M$ states the total number of data points.

The predicted model and actual calculated values associated with the response variables for omitting the AY23 for both training and validation sets are demonstrated in Fig. 3 and Fig. 4 respectively.

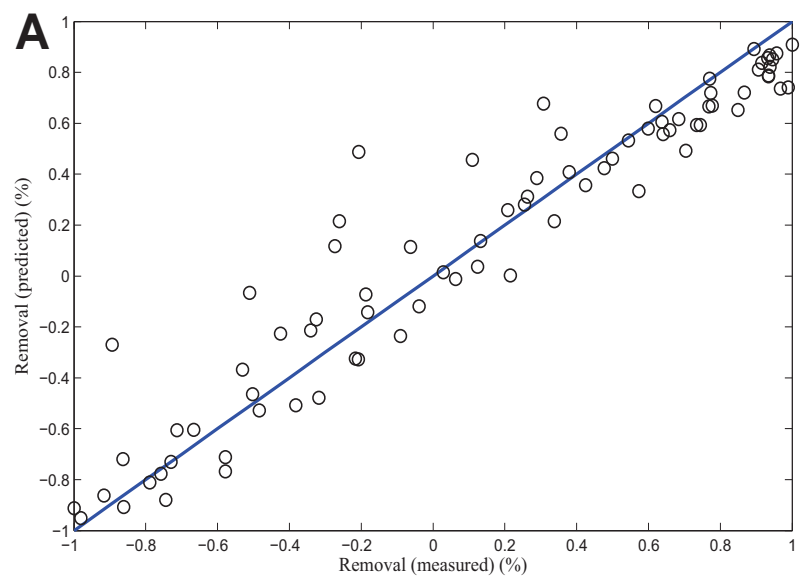

Fig.3. Calculated and predicted elimination of AY23 utilizing GA model in training set. 


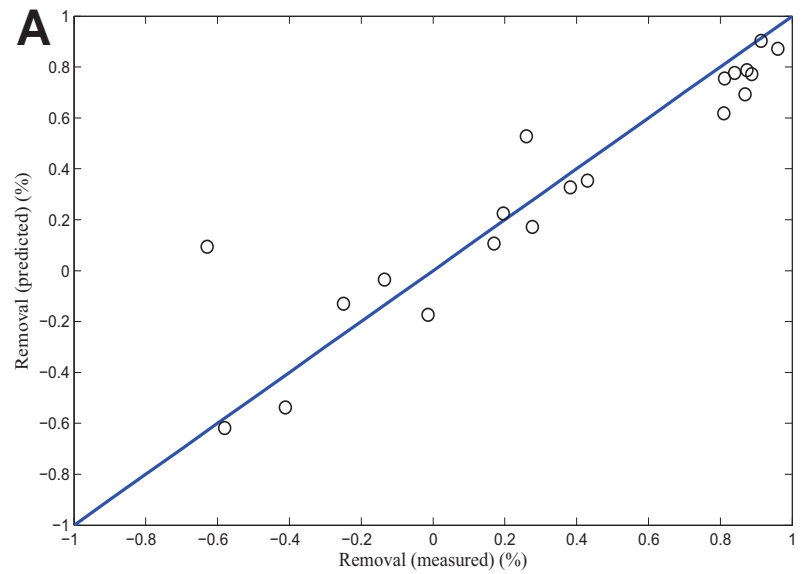

Fig.4. Calculated and predicted elimination of AY23 utilizing GA model in validation set.

The less amount of MSE produced by GA for both training and validation sets states the high performance of the model. In order to approximate the impact of each input on the output variable, the weight matrix is implemented. The following equation for connection weights is defined [40],

$$
A_{\iota}=\frac{\sum_{\alpha=1}^{\alpha=N_{\zeta}}\left(()\left|W_{\iota \alpha}^{\kappa \zeta}\right| / \sum_{\gamma=1}^{\gamma=N_{\kappa}}\left|W_{\gamma \alpha}^{\kappa \zeta}\right|\right) \times\left|W_{\alpha \beta}^{\zeta \nu}\right|}{\sum_{\gamma=1}^{\gamma=N_{\kappa}}\left\{\sum_{\alpha=1}^{\alpha=N_{\zeta}}\left(\left|W_{\gamma \alpha}^{\kappa \zeta}\right| / \sum_{\gamma=1}^{\gamma=N_{\kappa}}\left|W_{\gamma \alpha}^{\kappa \zeta}\right|\right) \times\left|W_{\alpha \beta}^{\zeta \nu}\right|\right\}}
$$

such that $A_{\iota}$ is considered as the relative significance of the $\iota$ th input variable on the output variable. $W \mathrm{~s}$ are considered as connection weights. $N_{\kappa}$ is taken to be the number of input neurons, and $N_{\zeta}$ is taken to be the number of hidden neurons. $\kappa, \zeta$ as well as $\nu$ states input, hidden and output layers, respectively. Subscripts $\gamma, \alpha$ and $\beta$ signifies input, hidden and output neurons, respectively.

\section{Conclusion}

In this work, GA procedure is proposed in order to predict the photocatalytic removal of AY23 by implementing the $\mathrm{Ag}-\mathrm{TiO}_{2}$ nanoparticles provided under appropriate conditions. The suggested technique is examined on 100 data set extracted from the literature. Experimental outcomes reveals that the GA technique is efficient for photocatalytic elimination of impurity in wastewater.

\section{References}

1. Chagas, E.P., Durrant, L.R.: Decolorization of azo dyes by phanerochate chrysosporium and pleurotus sajorcaju, Enzyme Microbial Technol, 29(1), 473-477 (2001). 
2. Fan, C.T., Wang, Y.K., Huang, C.R.: Heterogeneous information fusion and visualization for a large-scale intelligent video surveillance system, IEEE. Trans .Syst. Man, Cybern: Syst, 1-12 (2016).

3. Jafari, R., Razvarz, S.: Solution of fuzzy differential equations using fuzzy sumudu transforms, Mathematical and Computational Applications, 23, 1-15 (2018).

4. Jafari, R., Yu, W.: Uncertainty Nonlinear Systems Control with Fuzzy Equations, IEEE International Conference on Systems, Man, and Cybernetics, 28852890 (2015).

5. Jafari, R., Yu, W.: Artificial neural network approach for solving strongly degenerate parabolic and burgers-fisher equations, 12th International Conference on Electrical Engineering, Computing Science and Automatic Control, doi:10.1109/ICEEE.2015.7357914, (2015).

6. Jafari, R. Yu, W.: Uncertain nonlinear system control with fuzzy differential equations and Z-numbers, 18th IEEE International Conference on Industrial Technology, Canada, pp.890-895, doi:10.1109/ICIT.2017.7915477, (2017).

7. Jafari, R., Yu, W., Li, X.: Solving Fuzzy Differential Equation with Bernstein Neural Networks, IEEE International Conference on Systems, Man, and Cybernetics, Budapest, Hungary, 1245-1250 (2016).

8. S. Razvarz, R. Jafari, A. Gegov, W. Yu, S. Paul, Neural network approach to solving fully fuzzy nonlinear systems, Fuzzy modeling and control Methods Application and Research, Nova science publisher, Inc, New York. ISBN: 978-1-53613-415-5,4568 (2018).

9. Razvarz, S., Jafari, R., Granmo, O-C., Gegov, A.: Solution of dual fuzzy equations using a new iterative method, In Proceedings of the 10th Asian Conference on Intelligent Information and Database Systems, Lecture Notes in Artificial Intelligence (subseries of LNCS). Springer, 245-255 (2018).

10. Shirvani Ardekani, P., Karimi, H., Ghaedi, M., Asfaram, A., Kumar Purkait, M.: Ultrasonic assisted removal of methylene blue on ultrasonically synthesized zinc hydroxide nanoparticles on activated carbon prepared from wood of cherry tree: Experimental design methodology and artificial neural network, Journal of Molecular Liquids, 229, 114-124 (2017).

11. Mazaheri, H., Ghaedi, M., Ahmadi Azqhandi, M.H., Asfaram, A.: Application of machine/statistical learning, artificial intelligence and statistical experimental design for the modeling and optimization of methylene blue and $\mathrm{Cd}$ (II) removal from a binary aqueous solution by natural walnut carbon, Phys. Chem. Chem. Phys. 19, 11299-11317 (2017).

12. Chakraborty, P., Das, S., Roy, G.G., Abraham, A.: On convergence of the multiobjective particle swarm optimizers, Information Sciences, 181, 1411-1425 (2011).

13. El-Wakeel, A.S., Hassan, F., Kamel, A., Abdel-Hamed, A.: Optimum tuning of pid controller for a permanent magnet brushless DC motor, Int. J. Electr. Eng. Technol. 4, 53-64 (2013).

14. Fathi, V., Montazer, G.A.: An improvement in RBF learning algorithm based on PSO for real time applications, Neurocomputing. 111, 169-176 (2013).

15. Khajeh, M., Kaykhaii, M., Sharafi, A.: Application of PSO-artificial neural network and response surface methodology for removal of methylene blue using silver nanoparticles from water sample, J. Ind. Eng. Chem. http://dx.doi.org/10.1016/j.jiec.2013.01.033, (2013).

16. Eberhart, R.C.,Kennedy, J.: Swarm Intelligence, San Diego. CA: Morgan Kaufmann. (2001).

17. Kennedy, J., Eberhart, R.C.: Particle swarm optimization, In Proceedings of IEEE international conference on neural networks, Perth. Australia. 4, 1942-1948 (1995). 
18. Chen, C.L.P., Zhang, T., Tam, S.Ch.: A novel evolutionary algorithm solving optimization problems, IEEE International Conference on Systems, Man, and Cybernetics, San Diego, CA, USA, (2014).

19. Niknam,T., Taherian Fard, E., Pourjafarian, N., Rousta, A.: An efficient hybrid algorithm based on modified imperialist competitive algorithm and K-means for data clustering, Eng Appl Artif Intell. 24, 306-317 (2011).

20. Pothiya, S., Ngamroo, I., Kongprawechnon, W.: Application of multiple tabu search algorithm to solve dynamic economic dispatch considering generator constraints, Energy Conversion and Management. 49, 506-516 (2008).

21. Yousefi, M., Darus, A.N., Mohammadi, H.: An imperialist competitive algorithm for optimal design of plate-fin heat exchangers, Int $J$ Heat Mass Transfer. 55, 3178-3185 (2012).

22. Atashpaz-Gargari, E., Lucas, C.: Imperialist competitive algorithm: An algorithm for optimization inspired by imperialistic competition, IEEE Congress on Evolutionary Computation. Singapore, 4661-4667 (2007).

23. Azamuthullah, H.M., Ghani, A., Zakaria, N.A., Chang, C.K., Abu Hassan, Z.: Genetic programming approach to predict sediment concentration for Malaysian rivers, Interna. J. Ecologi. Econo. Statis. 16, 53-64 (2010).

24. Guven, A., Aytek, A.: New approach for stage-discharge relationship: gene expression programming, J. Hydro. Eng. 14, 812-820 (2009).

25. Sivanandam ,S.N., Deepa, S.N.: Introduction to Genetic Algorithms, Springer. New York. (2008).

26. Thomas, B.: Evolutionary algorithms in theory and practice: evolution strategies, evolutionary programming, genetic algorithms, Oxford University Press. (1996).

27. Goldberg, D.E.: Genetic algorithms in search optimization and machine learning, Reading, Massachusetts: Addison-Wesley. (1989).

28. Chang, Y., Erera, A.L., White, C.C.: Risk assessment of deliberate contamination of food production facilities, IEEE. Trans. Syst. Man, Cybern: Syst, 1-13 (2015).

29. Jafari, R., Razvarz, S.: Solution of Fuzzy Differential Equations using Fuzzy Sumudu Transforms, IEEE International Conference on Innovations in Intelligent Systems and Applications, 84-89 (2017).

30. Jafari, R., Razvarz, S., Gegov, A.: A New Computational Method for Solving Fully Fuzzy Nonlinear Systems, In: Computational Collective Intelligence. ICCCI 2018. Lecture Notes in Computer Science, Springer, Cham, 11055, 503-512 (2018).

31. Jafari, R., Razvarz, S., Gegov, A., Paul, S.: Fuzzy modeling for uncertain nonlinear systems using fuzzy equations and Z-numbers, Advances in Computational Intelligence Systems: Contributions Presented at the 18th UK Workshop on Computational Intelligence, September 5-7, 2018, Nottingham, UK. Advances in Intelligent Systems and Computing, Springer, 840, 66-107 (2018).

32. Jafari, R., Razvarz, S., Gegov, A., Paul, S., Keshtkar, S.: Fuzzy sumudu transform approach to solving fuzzy differential equations with z-numbers, $A d$ vanced Fuzzy Logic Approaches in Engineering Science. Hershey, PA: IGI Global, doi:10.4018/978-1-5225-5709-8.ch002, 18-48 (2018).

33. Jafari, R., Yu, W.: Fuzzy Modeling for Uncertainty Nonlinear Systems with Fuzzy Equations, Mathematical problems in Engineering. Vol.2017, https://doi .org/10.1155/2017/8594738, (2017).

34. Liang, G., Lan, X., Wang, J., Wang, J., Zheng, N.: A limb-based graphical model for human pose estimation, IEEE. Trans .Syst. Man, Cybern: Syst, 1-13 (2016).

35. Razvarz, S., Jafari, R.: Experimental study of al2o3 nanofuids on the thermal efficiency of curved heat pipe at different tilt angle, Journal of Nanomaterials, 1-7 (2018). 
36. Razvarz, S., Jafari, R.: Experimental Study of Al2O3 Nanofluids on the Thermal Efficiency of Curved Heat Pipe at Different Tilt Angle, 2nd international congress on technology engineering and science (ICONTES), Malaysia, (2016).

37. Razvarz, S., Jafari, R., Yu, W.: Numerical Solution of Fuzzy Differential Equations with Z-numbers using Fuzzy Sumudu Transforms, Advances in Science, Technology and Engineering Systems Journal (ASTESJ), 3, 66-75 (2018).

38. Razvarz, S., Vargas-Jarillo, C., Jafari, R., Gegov, A.: Flow Control of Fluid in Pipelines Using PID Controller, IEEE Access, 7, 25673-25680 (2019).

39. Yukalov, V.I., Sornette, D.: Quantitative predictions in quantum decision theory, IEEE. Trans .Syst. Man, Cybern: Syst, 1-15 (2016).

40. Kasiri, M.B., Aleboyeh, H., Aleboyeh, A.: Modeling and Optimization of Heterogeneous Photo-Fenton Process with Response Surface Methodology and Artificial Neural Networks, Environ. Sci. Technol. 42, 7970-7975, (2008). 